\title{
automatica
}

Automatica 39 (2003) 2025-2033

www.elsevier.com/locate/automatica

\section{Abstractions of Hamiltonian control systems ${ }^{\text {th }}$}

\author{
Paulo Tabuada*, George J. Pappas \\ Department of Electrical and Systems Engineering, 200 South 33rd Street, University of Pennsylvania, Philadelphia, PA 19104, USA
}

Received 30 January 2002; received in revised form 6 May 2003; accepted 3 July 2003

\begin{abstract}
Given a control system and a desired property, an abstracted system is a reduced system that preserves the property of interest while ignoring modeling detail. In previous work, abstractions of linear and nonlinear control systems were considered while preserving reachability properties. In this paper, we consider the abstraction problem for Hamiltonian control systems, where, in addition to the property of interest we also preserve the Hamiltonian structure of the control system. We show how the Hamiltonian structure of control systems can be exploited to simplify the abstraction process. We then focus on local accessibility preserving abstractions, and provide conditions under which local accessibility properties of the abstracted Hamiltonian system are equivalent to the local accessibility properties of the original Hamiltonian control system.
\end{abstract}

(C) 2003 Elsevier Ltd. All rights reserved.

Keywords: Nonlinear control systems; Hamiltonian control systems; Abstractions

\section{Introduction}

Property preserving abstractions of control systems are important for reducing the complexity of their analysis or design. From an analysis perspective, given a large-scale control system and a property to be verified, one extracts a smaller abstracted system with equivalent properties. Checking the property on the abstraction is then equivalent to checking the property on the original system. From a design perspective, rather than designing a controller for the original large-scale system, one designs a controller for the smaller abstracted system, and then refines the design to the original system while incorporating modeling detail.

This approach critically depends on whether we are able to construct hierarchies of abstractions as well as characterize conditions under which various properties of interest propagate from the original to the abstracted system and vice versa. In Pappas, Lafferriere, and Sastry (2000), hierarchical

\footnotetext{
This paper was not presented at any IFAC meeting. This paper was recommended for publication in revised form by Associate Editor Daizhan Cheng under the direction of Editor Hassan Khalil. This research is partially supported by DARPA under grant F33615-00-C-1707, and by Fundação para a Ciência e Tecnologia under grant PRAXIS $\mathrm{XXI} / \mathrm{BD} / 18149 / 98$.

${ }^{*}$ Corresponding author. Tel.: 215-746-2857; fax: 215-573-2068.

E-mail addresses: tabuadap@seas.upenn.edu (P. Tabuada), pappasg@seas.upenn.edu (G.J. Pappas).
}

abstractions of linear control systems were extracted using computationally efficient constructions, and conditions under which controllability of the abstracted system implied controllability of the original system were obtained. This led to extremely efficient hierarchical controllability algorithms. In the same spirit, abstractions of nonlinear control affine systems were considered in Pappas and Simic (2002), and the canonical construction for linear systems was generalized to nonlinear control affine systems. In Tabuada and Pappas (2001) existence of abstractions (regarded as quotients in a category) was shown for fully nonlinear control systems. Furthermore, a characterization of the relation between the state/input space of the original system with the state/input space of its abstraction was also presented.

In this paper, we proceed in the spirit of Pappas and Simic (2002), and consider abstractions of Hamiltonian control systems, which are control systems completely specified by controlled Hamiltonians. This additional structure allows to simplify the abstraction process by working with functions instead of vector fields or distributions as is the case for general nonlinear systems in Pappas and Simic (2002). This is possible since the controlled Hamiltonian contains all the relevant information that must be captured by the abstracted system. On the other hand, to be able to relate the dynamics induced by the controlled Hamiltonians, we need to restrict the class of abstracting maps to those that preserve the 
Hamiltonian structure. More precisely, given a Hamiltonian control system on a Poisson manifold $M$, and a (quotient) Poisson map $\phi: M \rightarrow N$, we present a canonical construction that extracts an abstracted Hamiltonian control system on $N$. We then characterize abstracting maps for which the original and abstracted system are equivalent from a local accessibility point of view.

Reduction of mechanical dynamical systems is a very rich and mature area and we point the reader to the standard references (Marsden \& Weinstein, 1974; Marsden \& Ratiu, 1986). Similar ideas were applied to mechanical control systems (de Alvarez, 1989; Krishnaprasad, 1985; Sreenath, Oh, Krishnaprasad, \& Marsden, 1988; van der Schaft, 1981) including also nonholonomic constraints (Bloch, Krishnaprasad, Marsden, \& Murray, 1996; Koon \& Marsden, 1997). The approach presented in this paper is quite different from these established notions of reduction for mechanical systems. When performing an abstraction one is interested in ignoring irrelevant modeling details. In this spirit, one factors the original model by group actions that do not necessarily represent symmetries. Furthermore, the proposed abstraction methodology does not rely on the existence of a Lie group acting on the state space. This extra freedom when abstracting is balanced by the fact that information about the system is lost, whereas when reducing, using symmetries, no essential information is lost.

The structure of this paper is as follows: In Section 2 we review Poisson geometry and controlled Hamiltonian systems. Sections 3 and 4 contain the main contributions of the paper: in Section 3 a notion of abstraction is introduced for Hamiltonian control systems and an algorithm for its construction is given; while in Section 4 we present conditions for local accessibility equivalence between the original and the abstracted system. Section 5 illustrates the paper results on a spherical pendulum example and at Section 6 interesting topics for further research are discussed.

\section{Hamiltonian control systems on Poisson manifolds}

In this section we review some basic facts from Poisson geometry as well as Hamiltonian control systems, in order to establish consistent notation. The reader may wish to consult numerous books on these subjects such as da Silva (2001); Marsden and Ratiu (1999); Nijmeijer and van der Schaft (1995).

\subsection{Poisson geometry}

Hamiltonian control systems can be defined on either symplectic or Poisson manifolds, however for the purposes of this paper, it will be more natural to work within the Poisson context. A Poisson structure on a smooth manifold $M$ is a bilinear map:

$$
\{-,-\}: C^{\infty}(M) \times C^{\infty}(M) \rightarrow C^{\infty}(M)
$$

called Poisson bracket, satisfying the following identities for any $f, g, h \in C^{\infty}(M)$ :

$$
\begin{aligned}
& \{f, g\}=-\{g, f\}, \\
& \{f,\{g, h\}\}+\{g,\{h, f\}\}+\{h,\{f, g\}\}=0, \\
& \{f g, h\}=\{f, h\} g+f\{g, h\} .
\end{aligned}
$$

A Poisson manifold is a smooth manifold $M$ equipped with a Poisson structure. Given a smooth function $h: M \rightarrow \mathbb{R}$, the Poisson bracket allows us to obtain a Hamiltonian vector field $X_{h}$ with Hamiltonian $h$ through the equality:

$\mathscr{L}_{X_{h}} f=\{f, h\} \quad \forall f \in C^{\infty}(M)$,

where $\mathscr{L}_{X_{h}} f$ is the Lie derivative of $f$ along $X_{h}$. Note that the vector field $X_{h}$ is well defined since the Poisson bracket satisfies the Leibnitz rule and therefore defines a derivation on $C^{\infty}(M)$ (Marsden \& Ratiu, 1999). Furthermore $C^{\infty}(M)$ equipped with a Poisson bracket is a Lie algebra, called a Poisson algebra.

As an example of a Poisson bracket consider $M=\mathbb{R}^{4}$ with coordinates $\left(q_{1}, q_{2}, p_{1}, p_{2}\right)$. We can define a Poisson bracket through the expression:

$$
\{f, g\}=\frac{\partial f}{\partial q_{1}} \frac{\partial g}{\partial p_{1}}+\frac{\partial f}{\partial q_{2}} \frac{\partial g}{\partial p_{2}}-\frac{\partial f}{\partial p_{1}} \frac{\partial g}{\partial q_{1}}-\frac{\partial f}{\partial p_{2}} \frac{\partial g}{\partial q_{2}} .
$$

Given now a smooth Hamiltonian $h: M \rightarrow \mathbb{R}$, we use (2.5) to define the corresponding Hamiltonian vector field $X_{h}$ through relation (2.4):

$$
\begin{aligned}
& \dot{q}_{1}=\mathscr{L}_{X_{h}} q_{1}=\left\{q_{1}, h\right\}=\frac{\partial h}{\partial p_{1}}, \\
& \dot{q}_{2}=\mathscr{L}_{X_{h}} q_{2}=\left\{q_{2}, h\right\}=\frac{\partial h}{\partial p_{2}}, \\
& \dot{p}_{1}=\mathscr{L}_{X_{h}} p_{1}=\left\{p_{1}, h\right\}=-\frac{\partial h}{\partial q_{1}}, \\
& \dot{p}_{2}=\mathscr{L}_{X_{h}} p_{2}=\left\{p_{2}, h\right\}=-\frac{\partial h}{\partial q_{2}} .
\end{aligned}
$$

We thus see that a Poisson bracket provides a geometric, coordinate free way of describing the Hamiltonian dynamics.

We also introduce some additional notation used throughout the paper. Given a smooth map $f: M \rightarrow N$ we denote by $T f: T M \rightarrow T N$ the tangent map of $f$, taking tangent vectors $X(x) \in T_{x} M$ at $x \in M$ to tangent vectors $T_{x} f \cdot X(x) \in T_{f(x)} N$ at $f(x) \in N$. We denote by $\mathbf{d} g$ the exterior derivative of a smooth map $g: N \rightarrow \mathbb{R}$ and define the pullback of $\mathbf{d} g$ by $f$, denoted by $f^{*} \mathbf{d} g$, as

$$
\left(f^{*} \mathbf{d} g\right)(x)(X)=\mathbf{d} g(f(x))\left(T_{x} f \cdot X\right) \quad \forall X \in T M .
$$

When $f$ is in fact a diffeomorphism, that is, a smooth map with a smooth inverse, we also define the pullback of a 
vector field $Y \in T N$ to be

$\left(f^{*} Y\right)(x)=T_{f(x)}\left(f^{-1}\right) \cdot Y \circ f(x)$.

Associated with the Poisson bracket there is a contravariant anti-symmetric two tensor

$B: T^{*} M \times T^{*} M \rightarrow \mathbb{R}$

such that

$B(x)(\mathbf{d} f, \mathbf{d} g)=\{f, g\}(x)$.

We say that the Poisson structure is nondegenerate if the map $B^{\#}: T^{*} M \rightarrow T M$ defined by

$\mathbf{d} g\left(B^{\#}(x)(\mathbf{d} f)\right)=B(x)(\mathbf{d} f, \mathbf{d} g)$

is an isomorphism for every $x \in M$.

Given a map $\phi: M \rightarrow N$ between Poisson manifolds, we say that $\phi$ preserves the Poisson structure or that $\phi$ is a Poisson map if

$\{f \circ \phi, g \circ \phi\}_{M}=\{f, g\}_{N} \circ \phi$

for every $f, g \in C^{\infty}(N)$.

\subsection{Hamiltonian control systems}

In this paper, we will focus on affine Hamiltonian control systems that we now define:

Definition 2.1. An affine Hamiltonian control system $\Sigma=(U, M, H)$ consists of a smooth manifold $U$ (the input space), a Poisson manifold $M$ with nondegenerate Poisson bracket (the state space), and a smooth function $H: M \times U \rightarrow \mathbb{R}$ (the controlled Hamiltonian). Furthermore, $H$ is locally of the form $H=h_{0}+\sum_{i=1}^{n} h_{i} u_{i}$, with $h_{i}$ locally defined smooth real valued maps and $\left(u_{1}, u_{2}, \ldots, u_{n}\right)$ local coordinates for $U$.

Using the controlled Hamiltonian and the Poisson structure on $M$ we can recover the familiar system map $F: M \times$ $U \rightarrow T M$ which is locally given by

$F=X_{h_{0}}+\sum_{i=1}^{n} X_{h_{i}} u_{i}$

and defines an affine distribution on $M$ given by

$\mathscr{D}_{M}(x)=X_{h_{0}}(x)+\operatorname{span}\left\{X_{h_{1}}(x), X_{h_{2}}(x), \ldots, X_{h_{n}}(x)\right\}$.

This distribution captures all the possible directions of motion available at a certain point $x$, and therefore describes a control system, up to a parameterization by control inputs. This affine distribution will be the object of interest throughout the remaining paper, and we will assume that the rank of $\mathscr{D}_{M}$ does not change with $x$. Furthermore, we will denote an affine distribution $\mathscr{D}_{M}$ by $X+\Delta$, where $X$ is a vector field and $\Delta$ a distribution. When this affine distribution is defined by a Hamiltonian control system we have $X=X_{h_{0}}$ and $\Delta=\operatorname{span}\left\{X_{h_{1}}, X_{h_{2}}, \ldots, X_{h_{n}}\right\}$. A similar reasoning is possible at the level of Hamiltonians. Locally, we can define the following affine space of smooth maps:

$\mathscr{H}_{M}=h_{0}+\operatorname{span}_{\mathbb{R}}\left\{h_{1}, h_{1}, \ldots, h_{n}\right\}$

which defines $\mathscr{D}_{M}$ by the equality:

$\mathscr{D}_{M}(x)=B^{\#}(x)\left(\mathbf{d} \mathscr{H}_{M}(x)\right)$.

Here we used the notation $\mathbf{d} \mathscr{H}_{M}$ to denote the set $\cup_{h \in \mathscr{H}_{M}} \mathbf{d} h$. We will also use the notation $\mathscr{H}_{M}=h_{0}+H_{\Delta}$ for an affine space of smooth maps where $h_{0}$ is a smooth map and $H_{\Delta}$ a linear space of smooth maps.

Having defined Hamiltonian control systems we turn to their trajectories or solutions:

Definition 2.2. A smooth curve $c: I \rightarrow M, 0 \in I \subseteq \mathbb{R}$ is called a trajectory of control system $\Sigma=(U, M, H)$, if there exists a curve $c^{U}: I \rightarrow U$ satisfying:

$\frac{\mathrm{d}}{\mathrm{d} t} c(t)=F\left(c(t), c^{U}(t)\right)$

for every $t \in I$.

\section{Abstractions of Hamiltonian control systems}

Given a Hamiltonian control system $\Sigma_{M}$ on a manifold $M$ and a map $\phi: M \rightarrow N$, our goal is to determine a new Hamiltonian control system $\Sigma_{N}$ on the manifold $N$ having as trajectories $\phi\left(c_{M}\right)$, where $c_{M}$ are trajectories of $\Sigma_{M}$. This new control system $\Sigma_{N}$ can be regarded as an abstraction of $\Sigma_{M}$ if the manifold $N$ is lower dimensional than $M$. In this case we are reducing the dimension of the control system, although capturing the relevant properties of the trajectories of $\Sigma_{M}$ through the map $\phi$. The choice of the map $\phi$ is problem dependent and reflects the knowledge one has about the system being analyzed. The following definition captures precisely this idea:

Definition 3.1. Let $\Sigma_{M}$ and $\Sigma_{N}$ be two Hamiltonian control systems on manifolds $M$ and $N$, respectively and $\phi: M \rightarrow N$ a Poisson map. Control system $\Sigma_{N}$ is a $\phi$-abstraction of control system $\Sigma_{M}$ iff for every trajectory $c_{M}$ of $\Sigma_{M}, \phi\left(c_{M}\right)$ is a trajectory of $\Sigma_{N}$.

From the above definition it is clear that an abstraction captures all the trajectories of the original system. It is this relation between trajectories that will allow us to relate properties of control systems with properties of its abstractions. Clearly, it is difficult to determine whether a control system is an abstraction of another at the level of trajectories. One is then interested in a characterization of abstractions which is equivalent to Definition 3.1 but algebraic. Such a description is given in the next result adapted from Pappas et al. (2000). 
Theorem 3.2. Let $\Sigma_{M}$ and $\Sigma_{N}$ be two Hamiltonian control systems defined on manifolds $M$ and $N$, respectively and $\phi: M \rightarrow N$ a Poisson map. Control system $\Sigma_{N}$ is a $\phi$-abstraction of $\Sigma_{M}$ if and only if $\Sigma_{N}$ is $\phi$-related to $\Sigma_{M}$, that is

$T_{x} \phi\left(\mathscr{D}_{M}(x)\right) \subseteq \mathscr{D}_{N} \circ \phi(x) \quad \forall x \in M$.

Making use of the above result we see that the notion of $\phi$-related control system is a generalization of the notion of $\phi$-related vector fields commonly found in differential geometry (Abraham, Marsden, \& Ratiu, 1988). This justifies the name of $\phi$-related control systems.

\subsection{Computing $\phi$-related control systems}

Given a Poisson map, Definition 3.1 provides us with a geometric definition for Hamiltonian abstractions which is useful conceptually but not computationally. We now present a canonical construction that will allow us to construct an abstraction $\Sigma_{N}$ from an Hamiltonian control system $\Sigma_{M}$ and a Poisson map $\phi: M \rightarrow N$. From this point on, we will work locally and assume that the map $\phi$ is a surjective, Poisson submersion with connected fibers, that is, the submanifolds $\phi^{-1}(y) \subseteq M$ are connected for every $y \in N$.

The construction to be presented is similar, in spirit, to the canonical construction in Pappas and Simic (2002), even though we work with Hamiltonians as opposed to distributions. This is natural for Hamiltonian systems since the differentials of the Hamiltonians capture all system information, that is, every $X_{h}$ in a basis of $\mathscr{D}_{M}$ is uniquely determined from $h$, given the Poisson structure.

Given a distribution $\mathscr{D} \subseteq T M$ we say that $\mathscr{D}$ is invariant under a vector field $Z \in T M$ when $\gamma_{t}^{*}(\mathscr{D}) \subseteq \mathscr{D}$ for every $t \in \mathbb{R}$ such that the flow $\gamma_{t}: M \rightarrow M$ of $Z$ is defined. This concept naturally extends to invariance under a distribution $\Delta$ by requiring $\mathscr{D}$ to be invariant under every vector field belonging in $\Delta$. Invariant affine distributions can alternatively be characterized as follows:

Proposition 3.3 (Pappas \& Simic, 2002). An affine distribution $\mathscr{D}=X+\Delta$ on $M$ is invariant under a vector field $Z$ iff for every $Y \in \mathscr{D}$ :

$[Z, Y] \subseteq \Delta$.

When the affine distribution of interest $\mathscr{D}_{M}$ is invariant under the distribution $\operatorname{Ker}(T \phi)$, it is shown in Pappas and Simic (2002) that $\mathscr{D}_{M}$ satisfies the equality:

$T_{x} \phi\left(\mathscr{D}_{M}(x)\right)=T_{x^{\prime}} \phi\left(\mathscr{D}_{M}\left(x^{\prime}\right)\right)$

for every $x^{\prime} \in \phi^{-1} \circ \phi(x)$. This provides a simple method to compute a $\phi$-related control system defined by an affine distribution $\mathscr{D}_{N}$ on $N$. To obtain $\mathscr{D}_{N}$ at a particular $y=\phi(x)$, it suffices to compute $T_{x} \phi\left(\mathscr{D}_{M}(x)\right)$ for some $x \in \phi^{-1}(y)$ in virtue of (3.3). This is, in fact, the rationale behind the construction given in Pappas and Simic (2002). We now exploit the same idea, although we will work at the level of
Hamiltonians. We start with an affine distribution defined by an affine space of Hamiltonians $\mathscr{H}_{M}=h_{0}+H_{\Delta}$ and extend it to ensure that it defines a new affine distribution invariant under $\operatorname{Ker}(T \phi)$. This new affine space of Hamiltonians will allow one to determine the desired affine space of Hamiltonians on $N$ by the use of a local section of $\phi$.

Definition 3.4 (Canonical construction). Let $\phi: M \rightarrow N$ be a Poisson map between manifolds with nondegenerate Poisson brackets, $\mathscr{H}_{M}=h_{0}+H_{\Delta}$ a locally defined affine space of Hamiltonians, $\mathscr{G}=\operatorname{span}_{\mathbb{R}}\left\{g_{1}, g_{2}, \ldots, g_{k}\right\}$ a locally defined linear space of Hamiltonians such that $\operatorname{Ker}(T \phi)=B_{M}^{\#}(\mathrm{~d} \mathscr{G})=\operatorname{span}\left\{X_{g_{1}}, X_{g_{2}}, \ldots, X_{g_{k}}\right\}$, and $\overline{\mathscr{H}_{M}}$ the following affine space of smooth maps:

$\overline{\mathscr{H}_{M}}=h_{0}+\overline{H_{\Delta}}+\left\{\overline{H_{\Delta}}, \mathscr{G}\right\}+\left\{\left\{\overline{H_{\Delta}}, \mathscr{G}\right\}, \mathscr{G}\right\}+\cdots$

with $\overline{H_{\Delta}}=H_{\Delta}+\left\{h_{0}, \mathscr{G}\right\}$. For any locally defined inclusion $i: N \rightarrow M$ satisfying $\phi \circ i=i d_{N}$ and $\mathrm{d} \overline{\mathscr{H}_{M}}(x)-$ $\phi^{*} i^{*} \mathrm{~d} \overline{\mathscr{H}_{M}}(x) \subseteq \mathrm{d} \mathscr{G}(x)$ for $x=i \circ \phi(x)$, the affine space of Hamiltonians $\mathscr{H}_{N}$ defined by

$\mathscr{H}_{N}=\overline{\mathscr{H}_{M}} \circ i$

is called canonically $\phi$-related to $\mathscr{H}_{M}$.

Remark 3.5. We note that the above construction can also be performed without using a basis of Hamiltonian vector fields for $\operatorname{Ker}(T \phi)$. Any other basis for $\operatorname{Ker}(T \phi)$ will equally work if we replace (3.4) with

$\overline{\mathscr{H}_{M}}=h_{0}+\overline{H_{\Delta}}+\mathscr{L}_{X} \overline{H_{\Delta}}+\mathscr{L}_{X} \mathscr{L}_{X} \overline{H_{\Delta}}+\cdots$

for every $X$ in the chosen basis of $\operatorname{Ker}(T \phi)$. This allows to compute $\overline{\mathscr{H}_{M}}$ even if $\mathscr{G}$ is not available. Nevertheless, it can be shown that, locally, such family $\mathscr{G}$ always exists.

Remark 3.6. In practice, the condition

$\mathrm{d} \overline{\mathscr{H}_{M}}(x)-\phi^{*} i^{*} \mathrm{~d} \overline{\mathscr{H}_{M}}(x) \subseteq \mathrm{d} \mathscr{G}(x), \quad x=i \circ \phi(x)$

may be replaced by the stronger condition

$\mathrm{d} h(x)-\phi^{*} i^{*} \mathrm{~d} h(x) \in \mathrm{d} \mathscr{G}(x), \quad x=i \circ \phi(x) \quad \forall h \in C^{\infty}(M)$

which is easier to check.

The control system defined by $B_{M}^{\#}\left(\mathbf{d} \overline{\mathscr{H}_{M}}\right)$ enjoys some properties which justify the use of the word canonical in Definition 3.4.

Proposition 3.7. The affine distribution $\overline{\mathscr{D}_{M}}=B_{M}^{\#}\left(\mathbf{d} \overline{\mathscr{H}_{M}}\right)$ is the smallest affine distribution, invariant under $\operatorname{Ker}(T \phi)$ which contains $\mathscr{D}_{M}$.

Proof. The distribution $\overline{\mathscr{D}_{M}}$ contains $\mathscr{D}_{M}$ since $\mathscr{H}_{M} \subseteq \overline{\mathscr{H}_{M}}$ and is affine by construction. Invariance under $\operatorname{Ker}(T \phi)$ follows from the equality $\{h, g\}=-\left[X_{h}, X_{g}\right]$ and Proposition 3.3. To show that $\overline{\mathscr{D}_{M}}$ is the smallest affine distribution with 
these properties we consider any other affine distribution $\mathscr{E}$ with the same properties. We now show that every vector field in $\overline{\mathscr{D}_{M}}$ also belongs to $\mathscr{E}$. Recall that, by construction, any vector field in $\overline{\mathscr{D}_{M}}$ can be written as a linear combination of vectors of the form

$Y=\sum_{j=0}^{k} a d_{X_{g(j)}^{j}}^{j} X$

for some $X \in \mathscr{D}_{M}, X_{g(j)} \in\left\{X_{g_{1}}, X_{g_{2}}, \ldots, X_{g_{k}}\right\}$ and $a d_{X_{g(j)}^{j}}^{j} X$ defined by

$a d_{X_{g(0)}}^{0} X=X$,

$a d_{X_{g(j+1)}}^{j+1} X=\left[a d_{X_{g(j)}}^{j} X, X_{g(j+1)}\right]$.

In view of this, we now show that $Y \in \mathscr{E}$ by induction on the number $k$ of Lie bracket depth

If $k=0$ then $X \in \mathscr{D}_{M} \subseteq \mathscr{E}$.

If $k=m$ and $k>1$, then we can write $Y$ as $Y=Z+\left[Z, X_{g}\right]$ for some $Z \in \overline{\mathscr{D}_{M}}$ and some $X_{g} \in\left\{X_{g_{1}}, X_{g_{2}}, \ldots, X_{g_{k}}\right\}$. Clearly $Z$ has bracket depth $m-1$ so that by the induction hypothesis $Z \in \mathscr{E}$. However $\mathscr{E}$ is $\operatorname{Ker}(T \phi)$ invariant so that Proposition 3.3 implies that $Y=Z+\left[Z, X_{g}\right] \in \mathscr{E}$. Since every vector field in $\overline{\mathscr{D}_{M}}$ is a linear combination of vector fields belonging to $\mathscr{E}$, the desired inclusion $\overline{\mathscr{D}_{M}} \subseteq \mathscr{E}$ follows.

The control system defined by $\mathscr{D}_{N}$ is the minimal abstraction of control system defined by $\mathscr{D}_{M}$.

Proposition 3.8. The affine Hamiltonian control system $\Sigma_{N}$, obtained from $\Sigma_{M}$ by the canonical construction is the smallest Hamiltonian control system on $N$-related to $\Sigma_{M}$.

Proof. We start by showing that the Hamiltonian control system defined by $\mathscr{H}_{N}$ is $\phi$-related to the Hamiltonian control system defined by $\mathscr{H}_{M}$. By construction of $\overline{\mathscr{H}_{M}}$ we have

$\mathscr{D}_{M}=B_{M}^{\#}\left(\mathbf{d} \mathscr{H}_{M}\right) \subseteq B_{M}^{\#}\left(\mathbf{d} \overline{\mathscr{H}_{M}}\right)=\overline{D_{M}}$

so that it suffices to show that $T_{x} \phi\left(\overline{\mathscr{D}_{M}}(x)\right) \subseteq \mathscr{D}_{N} \circ \phi(x)$ for any $x \in M$. By Proposition 3.7, $\mathscr{D}_{M}$ is $\operatorname{Ker}(T \phi)$ invariant and since $\phi$ has connected fibers we conclude that $T_{x} \phi\left(\overline{\mathscr{D}_{M}}(x)\right)=$ $T_{x^{\prime}}\left(\overline{\mathscr{D}_{M}}\left(x^{\prime}\right)\right)$ for any $x, x^{\prime} \in M$ such that $\phi(x)=\phi\left(x^{\prime}\right)$. It then suffices to show that $T_{x} \phi\left(\overline{\mathscr{D}_{M}}(x)\right) \subseteq \mathscr{D}_{N} \circ \phi(x)$ holds for some $x^{\prime} \in \phi^{-1}(\phi(x))$. We will choose such $x^{\prime}$ to be the point satisfying $i \circ \phi\left(x^{\prime}\right)=x^{\prime}$ since by assumption we have $\mathbf{d} \overline{\mathscr{H}_{M}}\left(x^{\prime}\right)-\phi^{*} i^{*} \mathbf{d} \overline{\mathscr{H}}_{M}\left(x^{\prime}\right) \subseteq \mathbf{d} \mathscr{G}\left(x^{\prime}\right)$ and it follows that

$$
\begin{aligned}
& B_{M}^{\#}\left(\mathbf{d} \overline{\mathscr{H}_{M}}\right)\left(x^{\prime}\right)-B_{M}^{\#}\left(\phi^{*} i^{*} \mathbf{d} \overline{\mathscr{H}_{M}}\right)\left(x^{\prime}\right) \subseteq B_{M}^{\#}(\mathbf{d} \mathscr{G})\left(x^{\prime}\right) \\
& \quad \Rightarrow T_{x^{\prime}} \phi\left(B_{M}^{\#}\left(\mathbf{d} \overline{\mathscr{H}_{M}}\right)\left(x^{\prime}\right)\right)-T_{x^{\prime}} \phi\left(B_{M}^{\#}\left(\phi^{*} i^{*} \mathbf{d} \overline{\mathscr{H}_{M}}\right)\left(x^{\prime}\right)\right) \\
& \quad \subseteq T_{x^{\prime}} \phi\left(B_{M}^{\#}(\mathbf{d} \mathscr{G})\left(x^{\prime}\right)\right)
\end{aligned}
$$

$$
\begin{aligned}
& \Rightarrow T_{x^{\prime}} \phi\left(B_{M}^{\#}\left(\mathbf{d} \overline{\mathscr{H}_{M}}\right)\left(x^{\prime}\right)\right)=T_{x^{\prime}} \phi\left(B_{M}^{\#}\left(\phi^{*} i^{*} \mathbf{d} \overline{\mathscr{H}_{M}}\right)\left(x^{\prime}\right)\right) \\
& \Rightarrow T_{x^{\prime}} \phi\left(B_{M}^{\#}\left(\mathbf{d} \overline{\mathscr{H}_{M}}\right)\left(x^{\prime}\right)\right)=T_{x^{\prime}} \phi\left(B_{M}^{\#}\left(\phi^{*} \mathbf{d}\left(\overline{\mathscr{H}_{M}} \circ i\right)\right)\left(x^{\prime}\right)\right) \\
& \Rightarrow T_{x^{\prime}} \phi\left(B_{M}^{\#}\left(\mathbf{d} \overline{\mathscr{H}_{M}}\right)\left(x^{\prime}\right)\right)=B_{N}^{\#}\left(\mathbf{d}\left(\overline{\mathscr{H}_{M}} \circ i\right)\right)\left(\phi\left(x^{\prime}\right)\right) \\
& \Rightarrow T_{x^{\prime}} \phi\left(\overline{\mathscr{D}_{M}}\left(x^{\prime}\right)\right)=\mathscr{D}_{N} \circ \phi\left(x^{\prime}\right),
\end{aligned}
$$

where the second implication follows from $B_{M}^{\#}(\mathbf{d} \mathscr{G}) \subseteq$ $\operatorname{Ker}(T \phi)$ and the fourth follows from the Poisson nature of the map $\phi$. From (3.6) and (3.7) we now conclude

$T_{x} \phi\left(\mathscr{D}_{M}(x)\right) \subseteq \mathscr{D}_{N} \circ \phi(x)$

for any $x \in M$. It remains to show that $\mathscr{D}_{N}$ is the smallest affine distribution satisfying (3.8). Consider any other affine distribution $\mathscr{E}$ on $N$ satisfying $T \phi\left(\mathscr{D}_{M}\right) \subseteq$ $\mathscr{E} \circ \phi$. From $\mathscr{E}$ we build a new distribution $\overline{\mathscr{E}}$ on $M$ by $\overline{\mathscr{E}}=(T \phi)^{-1}(\mathscr{E})$, that is $\overline{\mathscr{E}}=\{X \in T M: T \phi \cdot X \in \mathscr{E}\}$. It is clear that $\mathscr{D}_{M} \subseteq \overline{\mathscr{E}}$ and it is also true that $\overline{\mathscr{E}}$ is $\operatorname{Ker}(T \phi)$ invariant since for any $x, x^{\prime} \in M$ such that $\phi(x)=\phi\left(x^{\prime}\right)$, $T_{x} \phi(\overline{\mathscr{E}}(x))=\mathscr{E} \circ \phi(x)=\mathscr{E} \circ \phi\left(x^{\prime}\right)=T_{x^{\prime}} \phi\left(\overline{\mathscr{E}}\left(x^{\prime}\right)\right)$. However, by Proposition 3.7, $\overline{\mathscr{D}}_{M}$ is the smallest $\operatorname{Ker}(T \phi)$ invariant distribution containing $\mathscr{D}_{M}$. It then follows that

$\overline{\mathscr{D}_{M}} \subseteq \overline{\mathscr{E}} \Rightarrow T \phi\left(\overline{\mathscr{D}_{M}}\right) \subseteq T \phi(\overline{\mathscr{E}}) \Rightarrow \mathscr{D}_{N} \circ \phi \subseteq \mathscr{E} \circ \phi$

which shows that $\mathscr{D}_{N}$ is, in fact, the smallest affine distribution satisfying (3.8).

The previous proposition is very important since it ensures that given any affine Hamiltonian control system $\Sigma_{M}$ on a manifold $M$, and any Poisson surjective submersion $\phi: M \rightarrow N$, with connected fibers, it is always possible to compute the corresponding abstraction. Furthermore, it also ensures, that the resulting abstraction is the smallest over all affine control systems on $N \phi$-related to $\Sigma_{M}$. This fact justifies the term canonical for the construction given in Definition 3.4.

\section{Local accessibility equivalence}

In addition to propagating trajectories and Hamiltonians from the original control system to the abstracted system, we will investigate how accessibility properties can be preserved in the abstraction process. We first review several (local) accessibility properties for control systems (Isidori, 1996; Jurdjevic, 1997; Nijmeijer \& van der Schaft, 1995).

Definition 4.1. Let $\Sigma_{M}$ be a control system on a smooth manifold $M$. For each $T>0$ and each $x \in M$, the set of points reachable from $x$ at time $T$ by trajectories which remain in an open set $V$ containing $x$ for $t \leqslant T$, denoted by $\operatorname{Reach}^{V}(x, T)$, is equal to the set of terminal points $c_{M}(T)$ of $\Sigma_{M}$ trajectories that originate at $x$ and that satisfy $c_{M}(t) \in V$, for all $t \leqslant T$. The set of points reachable from $x$ in $T$ or fewer units of time while remaining in $V$, denoted by $\operatorname{Reach}^{V}(x, \leqslant T)$ is given by $\operatorname{Reach}^{V}(x, \leqslant T)=\cup_{t \leqslant T} \operatorname{Reach}^{V}(x, T)$. 
Definition 4.2. A control system $\Sigma_{M}$ is said to be

- Locally accessible from $x$ if $\operatorname{Reach}^{V}(x, \leqslant T)$ contains a non-empty open set $O$ of $M$ for all open sets $V$ and all $T>0$.

- Locally accessible if it is locally accessible from all $x \in M$.

- Controllable if for any $x_{1}, x_{2} \in M$, there exists a $T \in \mathbb{R}$ and a trajectory $c_{M}(t)$ of $\Sigma_{M}$ such that $c_{M}(0)=x_{1}$ and $c_{M}(T)=x_{2}$.

These local accessibility properties can be characterized by simple rank conditions on the Poisson algebra generated ${ }^{1}$ by the affine space of Hamiltonians $\mathscr{H}_{M}$.

Proposition 4.3 (Accessibility rank conditions). Let $\Sigma_{M}$ be an Hamiltonian control system on a manifold $M$ of dimension $m$ and denote by $\mathscr{P}\left(\mathscr{H}_{M}\right)$ the Poisson algebra generated by the smooth maps in $\mathscr{H}_{M}$. Then:

- If $\operatorname{dim}\left(\mathrm{d} \mathscr{P}\left(\mathscr{H}_{M}(x)\right)\right)=m$, then control system $\Sigma_{M}$ is locally accessible at $x \in M$.

- If $\operatorname{dim}\left(\mathrm{d} \mathscr{P}\left(\mathscr{H}_{M}(x)\right)\right)=m$ for all $x \in M$, then control system $\Sigma_{M}$ is locally accessible.

- If $\operatorname{dim}\left(\mathrm{d} \mathscr{P}\left(\mathscr{H}_{M}(x)\right)\right)=m$ for all $x \in M, \mathscr{H}_{M}$ is symmetric, that is $h \in \mathscr{H}_{M} \Rightarrow-h \in \mathscr{H}_{M}$, and $M$ is connected, then control system $\Sigma_{M}$ is controllable.

We now determine under what conditions on the abstracting maps, local accessibility of the original system $\Sigma_{M}$ is equivalent to local accessibility of its canonical abstraction $\Sigma_{N}$. In particular, conditions to propagate accessibility from the abstracted system $\Sigma_{N}$ to the original system $\Sigma_{M}$, are given in the next result.

Theorem 4.4 (Local accessibility equivalence). Let $\Sigma_{M}$ be a Hamiltonian control system canonically $\phi$-related to Hamiltonian control system $\Sigma_{N}, \mathscr{G}=\operatorname{span}_{\mathbb{R}}\left\{g_{1}, g_{2}, \ldots, g_{k}\right\}$ a locally defined linear space of Hamiltonians such that $\operatorname{Ker}(T \phi)=B_{M}^{\#}(\mathbf{d} \mathscr{G})=\operatorname{span}\left\{X_{g_{1}}, X_{g_{2}}, \ldots, X_{g_{k}}\right\}$, and $\mathscr{P}\left(\mathscr{H}_{M}\right)$ the Poisson algebra generated by $\mathscr{H}_{M}$. If $\mathscr{G} \subseteq \mathscr{P}\left(\mathscr{H}_{M}\right)$ then $\Sigma_{M}$ is locally accessible (at $x$ ) if and only if $\Sigma_{N}$ is locally accessible $($ at $\phi(x))$.

Proof. If $\mathscr{D}_{M}$ is locally accessible (at $x$ ) then by Theorem $3.2, \mathscr{D}_{N}$ is also locally accessible (at $\phi(x)$ ).

To show the converse we note that the equality $\{f, g\}=$ $\left[X_{g}, X_{f}\right]$ implies $\operatorname{dim}\left(\mathbf{d} \mathscr{P}\left(\mathscr{H}_{M}(x)\right)\right)=\operatorname{dim}\left(\operatorname{Lie}\left(\mathscr{D}_{M}(x)\right)\right)$ where $\operatorname{Lie}\left(\mathscr{D}_{M}\right)$ denotes the Lie algebra generated by the vector fields in $\mathscr{D}_{M}$. In virtue of this, we start by showing the following equality between the Lie algebras generated by $\mathscr{D}_{M}$ and its canonical $\phi$-abstraction $\mathscr{D}_{N}$ :

$$
T_{x} \phi\left(\operatorname{Lie}\left(\mathscr{D}_{M}\right)(x)\right)=\operatorname{Lie}\left(\mathscr{D}_{N}\right) \circ \phi(x) \text {. }
$$

\footnotetext{
${ }^{1}$ The Poisson algebra generated by a set of $S$ smooth maps on $M$ is the smallest Poisson subalgebra of $C^{\infty}(M)$ containing $S$.
}

Recall that $\overline{\mathscr{D}_{M}}$ (defined in Proposition 3.7) is $\operatorname{Ker}(T \phi)$ invariant, therefore there exists a local basis for $\overline{\mathscr{D}_{M}}$ of Ker $(T \phi)$-invariant vector fields. Let us denote by $\overline{X_{0}}+$ $\left\{\overline{X_{1}}, \overline{X_{2}}, \ldots, \overline{X_{n}}\right\}$ this basis. Since every vector field $\bar{X}$ in this basis is $\operatorname{Ker}(T \phi)$ invariant, it is $\phi$-related to its projection $\bar{Y} \circ \phi=T \phi(\bar{X})$. It then follows that $\overline{X_{1}}$ and $\overline{X_{2}}$ being $\phi$-related to $\overline{Y_{1}}$ and $\overline{Y_{2}}$, respectively, implies that $\left[\overline{X_{1}}, \overline{X_{2}}\right]$ is also $\phi$-related to $\left[\overline{Y_{1}}, \overline{Y_{2}}\right]$ and an induction argument shows that

$T \phi\left(\operatorname{Lie}\left(\overline{\mathscr{D}_{M}}\right)\right)=\operatorname{Lie}\left(\mathscr{D}_{N}\right) \circ \phi$.

The assumption $\mathscr{G} \subseteq \mathscr{P}\left(\mathscr{H}_{M}\right)$ and the equality $\{f, g\}=$ $\left[X_{g}, X_{f}\right]$ imply

$\operatorname{Ker}(T \phi) \subseteq \operatorname{Lie}\left(\mathscr{D}_{M}\right)$

and by construction of $\overline{\mathscr{D}_{M}}$ we have $\operatorname{Lie}\left(\overline{\mathscr{D}_{M}}\right)=\operatorname{Lie}\left(\mathscr{D}_{M}\right)$ which combined with (4.2) gives (4.1). Using now (4.1) we conclude

$$
\begin{aligned}
\operatorname{dim}\left(\operatorname{Lie}\left(\mathscr{D}_{M}\right)(x)\right)= & \operatorname{dim}\left(T_{x} \phi\left(\operatorname{Lie}\left(\mathscr{D}_{M}\right)(x)\right)\right) \\
& +\operatorname{dim}(\operatorname{Ker}(T \phi)(x)) \\
= & \operatorname{dim}\left(\operatorname{Lie}\left(\mathscr{D}_{N}\right) \circ \phi(x)\right) \\
& +\operatorname{dim}(\operatorname{Ker}(T \phi)(x)) .
\end{aligned}
$$

It is now easy to see that if $\Sigma_{N}$ is locally accessible at $\phi(x)$, then $\operatorname{dim}\left(\mathbf{d} \mathscr{P}\left(\mathscr{H}_{N}\right) \circ \phi(x)\right)=\operatorname{dim}\left(\operatorname{Lie}\left(\mathscr{D}_{N}\right) \circ \phi(x)\right)=\operatorname{dim}(N)$ and $\operatorname{dim}\left(\mathbf{d} \mathscr{P}\left(\mathscr{H}_{M}\right)(x)\right)=\operatorname{dim}\left(\operatorname{Lie}\left(\mathscr{D}_{M}\right)(x)\right)=\operatorname{dim}(N)+$ $\operatorname{dim}(\operatorname{Ker}(T \phi)(x))=\operatorname{dim}(M)$ since $\phi$ is a submersion. Local accessibility (at $x)$ now follows from $\operatorname{dim}\left(\mathbf{d} \mathscr{P}\left(\mathscr{H}_{M}\right)(x)\right)=$ $\operatorname{dim}(M)$ and Proposition 4.3.

As an immediate corollary we have:

Corollary 4.5. Let $\Sigma_{M}$ be a Hamiltonian control system canonically $\phi$-related to Hamiltonian control system $\Sigma_{N}, \mathscr{G}=\operatorname{span}_{\mathbb{R}}\left\{g_{1}, g_{2}, \ldots, g_{k}\right\}$ a linear space of Hamiltonians such that $\operatorname{Ker}(T \phi)=B_{M}^{\#}(\mathrm{~d} \mathscr{G})=\operatorname{span}\left\{X_{g_{1}}, X_{g_{2}}, \ldots, X_{g_{k}}\right\}$, and $\mathscr{P}\left(\mathscr{H}_{M}\right)$ the Poisson algebra generated by $\mathscr{H}_{M}$. If $\mathscr{G} \subseteq \mathscr{P}\left(\mathscr{H}_{M}\right)$ and both $\mathscr{H}_{M}$ and $\mathscr{H}_{N}$ are symmetric and furthermore $M$ is connected then $\Sigma_{M}$ is controllable iff $\Sigma_{N}$ is controllable.

Theorem 4.4 provides moderate conditions to propagate accessibility properties in a hierarchy of abstractions. This allows for a hierarchical approach to the determination of accessibility properties. As long as there are control inputs on the abstracted control system $\Sigma_{N}$, the Poisson algebra $\mathscr{P}\left(\mathscr{H}_{N}\right)$ contains non-constant maps and consequently the system can be further abstracted to an equivalent system regarding local accessibility by the use of a map $\phi$ such that $\operatorname{Ker}(T \phi) \subseteq B_{N}^{\#}\left(\mathbf{d} \mathscr{P}\left(\mathscr{H}_{N}\right)\right)$.

\section{A spherical pendulum example}

As an illustrative example, consider the spherical pendulum as a fully actuated mechanical control system. This 


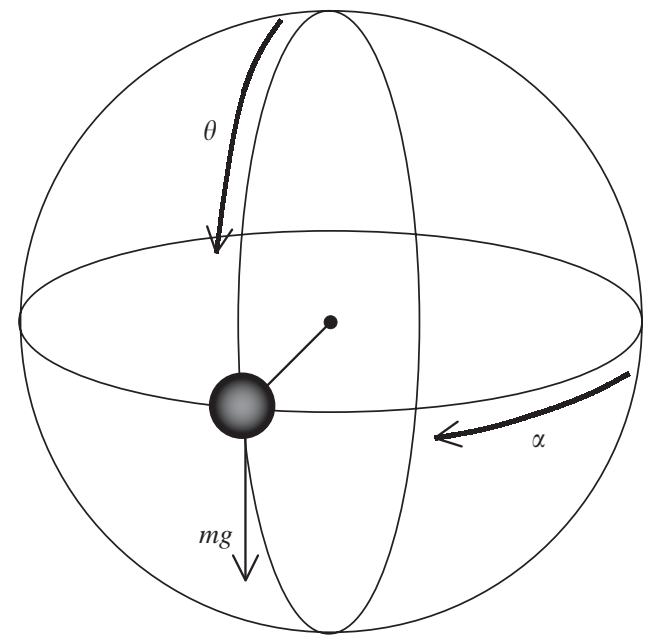

Fig. 1. Spherical pendulum with gravity force represented.

system can be used to model, for example, the stabilization of the spinning axis of a satellite or a pan and tilt camera. Consider a massless rigid rod of length $l$ fixed in one end by a spherical joint and having a bulb of mass $m$ on the other end. The configuration space for this control system is $S^{2}$, however we will work locally on $\left.C_{2} \subset S^{2}, C_{2}=\right] 0, \pi[\times] 0,2 \pi[$ parameterized by spherical coordinates $(\theta, \alpha)$, see Fig. 1 .

The kinetic energy of the system is given by

$T=\frac{1}{2} m l^{2}\left(\dot{\theta}^{2}+\sin ^{2} \theta \dot{\alpha}^{2}\right)$

and the potential energy of the system is

$V=-m g l \cos \theta$.

Through the Legendre transform of the Lagrangian $L=T-V$ one arrives at the Hamiltonian

$h_{0}=\frac{1}{2 m l^{2}} p_{\theta}^{2}+\frac{1}{2 m l^{2} \sin ^{2} \theta} p_{\alpha}^{2}-m g l \cos \theta$,

where $p_{\theta}$ is given by $p_{\theta}=m l^{2} \dot{\theta}$ and, $p_{\alpha}=m l^{2} \sin ^{2} \theta \dot{\alpha}$. Since the system is fully actuated, the controlled Hamiltonian defined over $M=T^{*} C_{2}$ is given by

$H_{M}=h_{0}+h_{1} u_{1}+h_{2} u_{2}$

with $h_{1}=\theta$ and $h_{2}=\alpha$ and, where $u_{1}$ and $u_{2}$ are the control inputs. Such controlled Hamiltonian defines control system $\Sigma_{M}$ through the use of the Poisson bracket:

$\{f, g\}=\frac{\partial f}{\partial \theta} \frac{\partial g}{\partial p_{\theta}}+\frac{\partial f}{\partial \alpha} \frac{\partial g}{\partial p_{\alpha}}-\frac{\partial f}{\partial p_{\theta}} \frac{\partial g}{\partial \theta}-\frac{\partial f}{\partial p_{\alpha}} \frac{\partial g}{\partial \alpha}$

as described in Section 2. The drift vector field associated with $h_{0}$ is invariant under rotations around the vertical axis and could be reduced using this symmetry by using the methods in Marsden and Ratiu (1986). However to emphasize the advantages of the abstraction method we will abstract away precisely the directions where there are no symmetries. Consider the abstracting map

$\phi: T^{*} C_{2} \rightarrow T^{*} C_{1}$,

$\left(\theta, \alpha, p_{\theta}, p_{\alpha}\right) \mapsto\left(\alpha, p_{\alpha}\right)$,

where $\left.C_{1}=\right] 0,2 \pi\left[\subset S^{1}\right.$ is equipped with the following Poisson bracket:

$\{f, g\}=\frac{\partial f}{\partial \alpha} \frac{\partial g}{\partial p_{\alpha}}-\frac{\partial f}{\partial p_{\alpha}} \frac{\partial g}{\partial \alpha}$.

If one lets $g_{1}=p_{\theta}, g_{2}=\theta$ and follow the steps of the canonical construction one obtains

$$
\begin{aligned}
& \left\{h_{0}, g_{1}\right\}=\frac{\partial h_{0}}{\partial \theta}=\frac{\cos \theta}{m l^{2} \sin ^{3} \theta} p_{\alpha}+m g l \sin \theta, \\
& \left\{\left\{h_{0}, g_{1}\right\}, g_{1}\right\}=\frac{\partial\left\{h_{0}, g_{1}\right\}}{\partial \theta}=-\frac{2+\cos 2 \theta}{m l^{2} \sin ^{4} \theta}+m g l \cos \theta, \\
& \left\{h_{0}, g_{2}\right\}=\frac{\partial h_{0}}{\partial p_{\theta}}=\frac{1}{m l^{2}} p_{\theta}, \\
& \overline{H_{\Delta}}=H_{\Delta}+\operatorname{span}_{\mathbb{R}}\left\{\left\{h_{0}, g_{1}\right\},\left\{h_{0}, g_{2}\right\}\right\} \\
& \quad=\operatorname{span}_{\mathbb{R}}\left\{\theta, \alpha, \frac{\cos \theta}{m l^{2} \sin ^{3} \theta} p_{\alpha}+m g l \sin \theta, \frac{1}{m l^{2}} p_{\theta}\right\} .
\end{aligned}
$$

Note that $\overline{H_{\Delta}}$ is of rank 4 except when $\theta=\pi / 2$, however by considering also $\left\{\left\{h_{0}, g_{1}\right\}, g_{1}\right\}$ we see that the affine distribution $\overline{H_{\Delta}}+\operatorname{span}_{\mathbb{R}}\left\{\left\{\left\{h_{0}, g_{1}\right\}, g_{1}\right\}\right\}$ has rank 4 on $C_{2}$. This implies that no further brackets need to be computed. Furthermore, we also see that $H_{\Delta}+\operatorname{span}_{\mathbb{R}}\left\{\left\{h_{0}, g_{1}\right\},\left\{h_{0}, g_{2}\right\}\right\}=$ $H_{\Delta}+\operatorname{span}_{\mathbb{R}}\left\{\left\{\left\{h_{0}, g_{1}\right\}, g_{1}\right\},\left\{h_{0}, g_{2}\right\}\right\}$ so that we will work with $\overline{\mathscr{H}_{M}}=H_{\Delta}+\operatorname{span}_{\mathbb{R}}\left\{\left\{\left\{h_{0}, g_{1}\right\}, g_{1}\right\},\left\{h_{0}, g_{2}\right\}\right\}$. The locally defined inclusion $i: N \rightarrow M$ mapping $\left(\alpha, p_{\alpha}\right)$ to $i\left(\alpha, p_{\alpha}\right)=$ $\left(\pi / 4, \alpha, 0, p_{\alpha}\right)$ satisfies $\phi \circ i=i d_{N}$. We now show that $i$ also satisfies $\mathbf{d} h-\phi^{*} i^{*} \mathbf{d} h \in \mathbf{d} \mathscr{G}$ for any $h \in C^{\infty}(M)$, as discussed in Remark 3.6. This follows from

$\phi^{*} i^{*} \mathbf{d} h=\mathbf{d}(h \circ i \circ \phi)=\frac{\partial h}{\partial \alpha} \mathbf{d} \alpha+\frac{\partial h}{\partial p_{\alpha}} \mathbf{d} p_{\alpha}$

which leads to $\mathbf{d} h-\phi^{*} i^{*} \mathbf{d} h=(\partial h / \partial \theta) \mathbf{d} \theta+\left(\partial h / \partial p_{\theta}\right) \mathbf{d} p_{\theta}=$ $(\partial h / \partial \theta) \mathbf{d} g_{2}+\left(\partial h / \partial p_{\theta}\right) \mathbf{d} g_{1} \in \mathbf{d} \mathscr{G}$.

The abstracted system is now obtained through $\mathscr{H}_{N}$ :

$$
\begin{aligned}
\mathscr{H}_{N}= & \overline{\mathscr{H}_{M}} \circ i \\
= & \frac{1}{m l^{2} \sin ^{2} \theta} p_{\alpha}^{2}-\frac{m g l}{\sqrt{2}} \\
& +\operatorname{span}_{\mathbb{R}}\left\{\frac{\pi}{3}, \alpha,-\frac{8}{m l^{2}} p_{\alpha}+\frac{m g l}{\sqrt{2}}\right\} .
\end{aligned}
$$

Discarding constant terms, which are associated with the zero vector field, we compute the abstraction to be

$\dot{\alpha}=\frac{2}{m l^{2}}\left(p_{\alpha}-4 v_{1}\right)$

$\dot{p}_{\alpha}=v_{2}$ 
which is a Hamiltonian control system on $N=T^{*} C_{1}$. Furthermore, since both $g_{1}$ and $g_{2}$ belong to $\mathscr{P}\left(\mathscr{H}_{M}\right)$ and $\Sigma_{N}$ is locally accessible, by making use of Theorem 4.4 we infer that $\Sigma_{M}$ is also locally accessible. This allows to use the abstracted model to do a preliminary control design to regulate the angle $\alpha$ on the smaller, abstracted model, which can be later extended to a complete design regulating both $\alpha$ and $\theta$. This has the advantage of breaking the design in two phases, where only one variable is regulated in each phase. In concrete applications one would build an hierarchy of abstractions, where Theorem 4.4 would be used to ensure that every control design at the upper levels can be implemented at the lower levels, therefore reducing the complexity of control design. Furthermore, since each level is an Hamiltonian control system, specific techniques for mechanical systems control design (Ortega, van der Schaft, Maschke, \& Escobar, 1999; Bloch, Leonard, \& Marsden, 2000; Bloch, Chang, Leonard, \& Marsden, 2001; Bullo \& Murray, 1999; Bullo, Leonard, \& Lewis, 2000) can be used at each level of design.

\section{Conclusions}

In this paper, we have presented a hierarchical abstraction methodology for affine Hamiltonian control systems. We presented an algorithm for its determination which computes the smallest abstraction based on a given abstraction map. All the computations are performed at the level of Hamiltonians which simplifies the process. We have also characterized local accessibility preserving maps. This allows to build an hierarchy of control systems with different dimensions but equivalent local accessibility properties. These results are very encouraging for hierarchical control of mechanical systems.

Refining controller design from the abstracted to the original system is clearly important and will certainly build on the recent results (Tabuada \& Pappas, 2001) describing how state/inputs of the original system are related to state/inputs of the abstracted system. Other research topics of great interest include the extension of present framework to port controlled Hamiltonian system (pcHs) and related notions of interconnection (van der Schaft \& Maschke, 1997) as well as the propagation of nonholonomic constraints in the context of pcHs.

\section{Acknowledgements}

The authors would like to acknowledge one of the anonymous reviewers for the careful and insightful comments which greatly improved the final manuscript.

\section{References}

Abraham, R., Marsden, J., \& Ratiu, T. (1988). Manifolds, tensor analysis and applications. Applied mathematical sciences. Berlin: Springer.
Bloch, A. M., Chang, D., Leonard, N. E., \& Marsden, J. E. (2001). Controlled Lagrangians and the stabilization of mechanical systems II: Potential shaping. IEEE Transactions on Automatics Control, 46(10), $1556-1571$

Bloch, A. M., Krishnaprasad, P. S., Marsden, J. E., \& Murray, R. (1996). Nonholonomic mechanical systems with symmetry. Archive for Rational Mechanics and Analysis, 136(1), 21-99.

Bloch, A. M., Leonard, N. E., \& Marsden, J. E. (2000). Controlled lagrangians and the stabilization of mechanical systems I: The first matching theorem. IEEE Transactions on Automatic Control, 45(12), 2253-2270.

Bullo, F., Leonard, N.E., \& Lewis, A.D. (2000). Controllability and motion algorithms for underactuated lagrangian systems on lie groups. IEEE Transactions on Automatic Control, 45(8), 1437-1454.

Bullo, F., \& Murray, R. M. (1999). Tracking for fully actuated mechanical systems: A geometric framework. Automatica, 35, 17-34.

da Silva, A. C. (2001). Lectures on symplectic geometry. In Lecture Notes in Mathematics, Vol. 1764. Berlin: Springer.

de Alvarez, G. S. (1989). Controllability of poisson control systems with symmetries. In: J. E. Marsden, P. S. Krishnaprasad, J. C. Simo (Eds.), Dynamics and control of multi-body system, Contemporary mathematics Vol. 97. Providence, RI: American Mathematical Society.

Isidori, A. (1996). Nonlinear control systems (3rd ed.). Berlin: Springer.

Jurdjevic, V. (1997). Geometric control theory. In Cambridge studies in advanced mathematics, Vol. 1. Cambridge: Cambridge University Press.

Koon, W. S., \& Marsden, J. E. (1997). Optimal control for holonomic and nonholonomic mechanical systems with symmetry and Lagrangian reduction. SIAM Journal on Control and Optimization, 35, 901-929.

Krishnaprasad, P. S. (1985). Lie-Poisson structures, dual-spin spacecraft and asymptotical stability. Archive for Rational Mechanics and Analysis, 9, 1011-1035.

Marsden, J., \& Ratiu, T. (1986). Reduction of Poisson manifolds. Letters in Mathematical Physics, 11, 161-170.

Marsden, J. E., \& Ratiu, T. S. (1999). Introduction to mechanics and symmetry. Texts in applied mathematics. Berlin: Springer.

Marsden, J. E., \& Weinstein, A. (1974). Reduction of symplectic manifolds with symmetry. Reports on Mathematical Physics, 5, $120-121$.

Nijmeijer, H., \& van der Schaft, A. J. (1995). Nonlinear dynamical control systems. Berlin: Springer.

Ortega, R., van der Schaft, A., Maschke, B., \& Escobar, G. (1999). Energy-shaping of port-controlled Hamiltonian systems by interconnection. In: Proceedings of the 37th IEEE conference on decision and control, Phoenix, AZ, pp. 1646-1651.

Pappas, G. J., Lafferriere, G., \& Sastry, S. (2000). Hierarchically consistent control systems. IEEE Transactions on Automatic Control, 45(6), $1144-1160$.

Pappas, G. J., \& Simic, S. (2002). Consistent hierarchies of affine nonlinear systems. IEEE Transactions on Automatic Control, 47(5), $745-756$.

Sreenath, N., Oh, Y. G., Krishnaprasad, P. S., \& Marsden, J. E. (1988). The dynamics of coupled planar rigid bodies, Part I: Reduction, equilibria and stability. Dynamics and Stability of Systems, 3, $25-49$.

Tabuada, P., \& Pappas, G. J. (2001). Quotients of fully nonlinear control systems. SIAM Journal on Control and Optimization. Under revision, available at www.seas.upenn.edu/ tabuadap.

van der Schaft, A. (1981). Symmetries and conservation laws for Hamiltonian systems with inputs and outputs: A generalization of Noether's theorem. Systems and Control Letters, 1, $108-115$.

van der Schaft, A. J., \& Maschke, B. (1997). Interconnected mechanical systems, Part I: Geometry of interconnection and implicit Hamiltonian systems. In: A. Astolfi, D. J. N. Limebeer, C. Melchiorri, A. Tornambe, 
R. B. Vinter (Eds.), Modelling and control of mechanical systems. London: Imperial College Press.

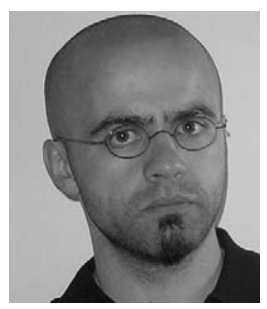

Paulo Tabuada was born in Lisbon, Portugal during the year of 1975 . He has received his "Licenciatura" degree in Aerospace Engineering from Instituto Superior Técnico, Lisbon, Portugal in 1998 and his Ph.D. degree in Electrical and Computer Engineering in 2002 from the Institute for Systems and Robotics, a private research institute associated with Instituto Superior Técnico. Between January 2002 and July 2003 he was a postdoctoral researcher at the University of Pennsylvania. He is currently an assistant professor in the Department of Electrical Engineering at the University of Notre Dame. Paulo Tabuada was the recipient of the Francisco de Holanda prize in 1998 for the best research project with an artistic or aesthetic component awarded by the Portuguese Science Foundation. He was a finalist for the Best Student Paper Award at both the 2001 American Control Conference and the 2001 IEEE Conference on Decision and Control and he was an Outstanding Reviewer for the IEEE Transactions on Automatic Control in 2002. His research interests include analysis and controller synthesis for discrete, timed, hybrid and embedded systems as well as geometrical control theory for nonlinear and Hamiltonian systems, hierarchical and distributed control systems.

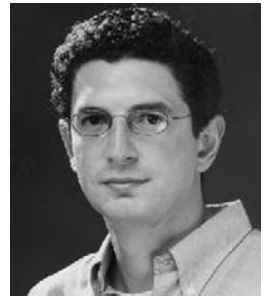

George J. Pappas received the B.S. degree in Computer and Systems Engineering in 1991, the M.S. degree in Computer and Systems Engineering in 1992, both from Rensselaer Polytechnic Institute, Troy, NY. In 1994, he was a Graduate Fellow at the Division of Engineering Science of Harvard University. In December 1998, he received the Ph.D degree from the Department of Electrical Engineering and Computer Sciences at the University of California at Berkeley. He was a postdoctoral researcher at the University of California at Berkeley and the University of Pennsylvania. His is currently an Assistant Professor and Graduate Group Chair in the Department of Electrical and Systems Engineering at the University of Pennsylvania, where he also holds a secondary appointment in the Department of Computer and Information Sciences. George Pappas is the recipient the NSF CAREER award in 2002, and the 1999 Eliahu Jury Award for Excellence in Systems Research from the Department of Electrical Engineering and Computer Sciences at the University of California at Berkeley. He was also a finalist for the Best Student Paper Award at the 1998 IEEE Conference on Decision and Control. His research interests include hierarchical control systems, embedded hybrid systems, distributed control systems, nonlinear control systems, geometric control theory, with applications to flight management systems, robotics, and unmanned aerial vehicles. 